\title{
CAPM - Markov Switching e Kalman Filter: uma Aplicação aos Indices Setoriais de Sustentabilidade e Governança da B3
}

\section{CAPM - Markov Switching and Kalman Filter: an Application to B3's Sustainability and Governance Industry Indices}

\author{
Ricardo de Souza Tavares ${ }^{\mathrm{a}}$ \\ João Frois Caldeira ${ }^{\mathrm{b}, \mathrm{c}}$
}

\begin{abstract}
Resumo: Este estudo busca investigar a formação dos retornos de índices setoriais e de práticas corporativas da B3. Para tanto, utiliza-se uma metodologia tradicional com os modelos de precificação de ativos de capital (CAPM) em suas versões estática - com mudança de regime (Markov switching) - e com betas variando a cada ponto do tempo - filtro e suavizador de Kalman. A aplicação dessa metologia traz evidências de que oito dos nove índices analisados apresentam mudança estrutural (alternam entre dois regimes). Além disso, nota-se que os betas são instáveis ao longo do tempo, isto é, há uma relação não linear entre risco e retorno. De modo geral, os resultados encontrados indicam que o risco sistêmico (beta) dos índices analisados variam ao longo do tempo e dependem de regimes. Por fim, a presente análise possibilita ao gestor ou investidor ter acesso a um conjunto de informações relevantes para sua tomada de decisão em relação a investimentos em setores ou conjunto de empresas com boas práticas que compõem a bolsa de valores brasileira.
\end{abstract}

Palavras-chave: CAPM. Ibovespa. Markov switching. Filtro de Kalman.

\begin{abstract}
This paper seeks to understand the behavior of the sectoral, governance and sustainability indices of the Brazilian stock exchange. For this, a traditional methodology was used with the CAPM models in their versions: static, with regime change (Markov Switching) and with betas varying at each point of time (Kalman Filter and Smoother). The application of this methodology brought evidence that eight of the nine indexes analyzed present structural change (alternating between two regimes). In addition, it was noted that betas are unstable over time, ie there is a nonlinear relationship between risk and return. Overall, the results found indicate that the systemic risk (beta) of the analyzed indices varies over time and depends on regimes. Finally, this analysis allows the manager or investor to have access to a set of information relevant to his decision

a University of Arizona, Eller College of Management, Department of Economics. Tucson, Arizona, United States of America.

b Universidade Federal de Santa Catarina, Departamento de Economia e Relações Internacionais. Florianópolis, Santa Catarina, Brasil.

c Conselho Nacional de Desenvolvimento Científico e Psicológico (CNPq). Brasília, Brasil.
\end{abstract}


making regarding investments in sectors or a set of companies with good practices, which make up the Brazilian stock exchange.

Keywords: CAPM. Ibovespa. Markov switching. Kalman filter.

JEL Classification: G10; G11; G12.

\section{1 lntrodução}

O mercado de capitais brasileiro vem se consolidando como um ambiente seguro e acessível ao investidor. Há mais de 1 milhão de investidores pessoa física operando no mercado de renda variável brasileiro. O principal índice do mercado, o Ibovespa, que pode ser interpretado com um termômetro para apuração do clima no ambiente de negócios, tem apresentado uma escalada recente, atingindo pela primeira na vez história níveis acima dos 100 mil pontos. Apesar do resultado ser estabelecido em termos nominais, é uma marca simbólica e demonstra o quanto o mercado de ações brasileiro tem despertado interesse.

Outro fato que tem alavancado os investimentos em renda variável fica por conta das sucessivas reduções observadas na taxa de juros (Selic) em períodos recentes, atingindo o menor nível desde a sua criação, 6 em julho de 2019. Nesse contexto de maior relevância do mercado de renda variável brasileiro, muitos investidores, especialmente em nível institucional, têm optado pela especialização na alocação de seus recursos.

Há uma vasta gama de fundos de investimentos que têm concentrado seus portfólios em setores específicos. A experiência e redução de custos de informação associadas ao conhecimento e atuação em determinando setor, ou conjunto específico de empresas, são fundamentais ao gestor em sua tomada de decisão em termos de alocação. De fato, o conhecimento e a visão do gestor num processo de construção de portfólio é de extrema importância quando se visa alcançar bons resultados em termos de retornos. Black e Litterman (1990) foram os pioneiros a incorporar a visão dos gestores na construção de carteiras teóricas.

Por outro lado, quando são observados mercados de renda variável mais consolidados, pode-se notar que o investidor institucional, ou seja, o investidor que busca retorno a longo prazo, está extremamente interessado em conhecer não só aspectos das empresas que detêm participação, como também do setor ao qual a empresa está inserida, ou, mesmo, de empresas com práticas gerenciais que se assemelham a sua. Um investidor que opera no setor elétrico, por exemplo, necessita manter-se informado sobre as politícas para o setor, condições de demanda, assim como características da formação de preços e retornos de ações de empresas que compõem o seu setor de atuação. 
Ainda em relação ao mercado brasileiro, foram construídos índices que sintetizam o comportamento de determinados setores econômicos ou de empresas que seguem determinadas práticas corporativas. Entre eles estão os índices setoriais - que são constituídos de carteiras teóricas que capturam, em média, a performance das ações de um conjunto de empresas de setores como de consumo, financeiro, imobiliário, entre outros -, além dos índices de sustentabilidade e governança - que sumarizam, respectivamente, o desempenho das ações de empresas com práticas sustentáveis e de governança corporativa que atendam a alguns requisitos predeterminados em sua construção.

Baca, Garbe e Weiss (2000) são autores que estudaram a composição dos retornos dos mercados setoriais de economias desenvolvidas. Eles encontraram evidências de que, no período recente à elaboração de seu estudo, os movimentos dos preços das ações, independente do país ao qual as empresas pertenciam, apresentavam uma determinação maior dada pelos índices setoriais, inclusive em termos globais, que os índices de mercado dos próprios países. Dito de outra forma, seriam mais importantes as transformações de um dado setor de forma global que a economia local, de modo geral, para determinação do comportamento dos ativos de um setor em questão.

Além dos índices setoriais, existem aqueles que agrupam empresas com boas práticas corporativas. Há estudos como os de Miles e Covin (2000), Plantinga e Scholtens (2001), McCool e Stankey (2004) e López, Garcia e Rodriguez (2007) que citam trabalhos ou fazem análises nas quais índices cuja carteira teórica é composta por ações de empresas com boas práticas sociais teriam desempenho superior aos respectivos índices de mercado. Para o caso brasileiro, há inúmeros trabalhos que analisam o retorno de índices de sustentabilidade, como os de Rezende, Nunes e Portela (2008) e Machado, Machado e Corrar (2009), além do de Milani et al. (2012), que estudam a volatilidade de índices de governança e sustentabilidade e concluem que, de modo geral, tendem a ser menos voláteis que os índices de mercado.

Quando se analisa o processo de formação dos índices setoriais ou de empresas com práticas de gestão similares, os modelos de precificação de ativos e suas diferentes abordagens têm grande espaço na literatura. De maneira geral, os modelos de precificação podem ser empregados com diversas finalidades, como testar a sensibilidade dos retornos dos ativos em relação ao retorno do mercado e estruturar essa relação ao longo do tempo, também podendo ser utilizados num contexto de previsão, além de auxiliar a estruturação de carteiras de investimento. Do ponto de vista do investidor, são ferramentas úteis que permitem identificar e mensurar as fontes de variação nos retornos dos ativos ou índices e, assim, auxiliam nas tomadas de decisões. Dessa forma, os modelos de precificação de ativos 
de capital (CAPM) são uma ferramenta muito popular e de fácil implementação computacional para problemas dessa natureza.

Os modelos CAPM são frequentemente abordados em trabalhos sobre mercado de capitais e econometria financeira. Campbell (1996) é um dos autores que traz uma discussão detalhada acerca deles. Em sua versão univariada mais simples, o modelo CAPM assume que os retornos dos ativos dependem linearmente dos retornos do mercado e de um ativo livre de risco. Essa abordagem permite o estudo do comportamento, em termos de risco e retorno esperados, de ativos individuais ou carteiras de ativos em relação ao mercado como um todo.

Dentro da abordagem CAPM, o parâmetro beta é utilizado de forma a capturar o risco sistêmico. Fama (1968), em um dos seus trabalhos seminais nesse campo, introduziu o símbolo como medida de sensibilidade do retorno de um ativo ao retorno de mercado. Desde então, no contexto de precificação de ativos, o parâmetro é utilizado sempre que há referência ao risco do mercado. A abordagem desse modelo mostra que a sensibilidade de um ativo (no caso são tomados ativos como índices) às variações dos retornos da carteira de mercado produz os retornos esperados de um ativo qualquer.

Entre as técnicas para estimação do modelo CAPM, abordagens que levam em conta as mudanças de regimes têm-se mostrado boas alternativas em problemas empíricos. Huang (2000) e Škrinjarić (2014) estão entre os autores que realizam a estimação com modelos Markov switching. Os autores utilizam técnicas desenvolvidas por Hamilton (1989) para incorporar mudanças nas variáveis observáveis, modelando não apenas os parâmetros das equações, mas também as probabilidades de se estar nos diferentes estados. A intuição econômica por trás da aplicação da abordagem Markov switching ao modelo CAPM pode ser orientada através da observação e detecção do mercado em seus dois estados levando em conta as volatidades bull e bear. Bull market é caracterizado como um regime de retorno positivo e baixa volatilidade, por outro lado, um regime com alta volatilidade e retorno negativo é definido como bear market. Também pode-se avaliar os regimes de acordo com o parâmetro $\beta$, como será feito aqui, em seus dois estados: de alavancagem ( $\beta$ alto) ou conservador ( $\beta$ baixo). Nesse caso, a amplitude do parâmetro $\beta$ diria o quão alavancados os retornos dos ativos estão em relação ao mercado.

Outra ferramenta indicada para se lidar com o problema da estimação dos coeficientes de risco sistêmico variando ao longo do tempo é o filtro Kalman, que possibilita, através da extração de componentes não observáveis, a construção de modelos dinâmicos com parâmetros que se alteram a cada ponto do tempo. Há uma série de trabalhos envolvendo a aplicação do filtro de Kalman em problemas com modelos de precificação, como os de Groenewold e Fraser (1999), Huang (2001), Petris et al. (2011), entre outros. 
Do ponto de vista do investidor, a abordagem de Markov switching permite analisar de forma mais objetiva o tempo no qual um ativo se porta de modo mais agressivo ou conservador em relação ao mercado (mudança de regime). Já o filtro de Kalman possibilita ao investidor observar como o retorno de um ativo portou-se em relação ao mercado a cada ponto do tempo, além de permitir a previsão um passo à frente para o comportamento do risco sistêmico e do retorno de qualquer ativo.

Não é raro que se queira ter informações acerca de portfólios industriais (ou índices que os representem), além de haver diversas aplicações em problemas financeiros, como seleção de carteiras, como pode ser visto no trabalho de Kirby e Ostdiek (2012), ou precificação de ativos, como descrito por Brooks, Faff e McKenzie (1998) e Gastaldi e Nardecchia (2003). No caso destes dois trabalhos, houve a estimação do modelo CAPM com os coeficientes de risco sistêmico condicionais. Desse modo, pretende-se analisar neste artigo o comportamento dos índices setoriais e de boas práticas gerenciais em relação aos movimentos do mercado de ações brasileiro, sintetizado pelo Ibovespa: empregando-se, nas estimações, as técnicas de Markov switching e filtro de Kalman, considerando-se a informação disponível até o presente (filtragem) e o caso no qual se conta com toda a informação de toda a amostra (suavização), de forma que seja possível conhecer mais acerca do processo de construção dos retornos desses índices com o intuito de se fornecer mais informações ao investidor ligado a um setor ou a um conjunto de empresas que adotem boas práticas institucionais.

Portanto, o objetivo deste trabalho é estimar a sensibilidade dos retornos (risco sistêmico) dos principais índices setoriais, de sustentabilidade e governança da bolsa de valores brasileira em relação ao retorno de mercado. Para isso, é importante ter-se em vista o fato de que a análise de precificação desses portfólios pode trazer inúmeros ganhos de informação ao agente em seu processo de tomada de decisões de investimento em relação a ativos dos diversos setores do mercado ações brasileiro, além de ser mais um experimento no qual se averígua a inconstância temporal do risco sistêmico, nesse caso para as carteiras industriais brasileiras.

A análise conta com observações semanais dos retornos dos índices setoriais e de práticas corporativas, do índice de mercado e do certificado de depósito interbancário (CDI) para 252 dias, que é tomado como proxy para o ativo livre de risco, para um período compreendido de 10 anos, que vai de 9 de maio de 2008 a 11 de maio de 2018. O índice Ibovespa é utilizado como proxy para o mercado. A definição do período foi realizada devido ao surgimento recente de vários dos índices em estudo, sendo que, a partir da data inicial, há dados para todos. Realizam-se estimações com o modelo em formato estático, em uma versão com mudança de regime, além do caso em que há variação a cada ponto do tempo através do filtro e da suavização de Kalman. 


\section{Modelo de Equilbrio de Mercado (CAPM)}

A partir das suposições clássicas do modelo de precificação de ativos ${ }^{1}$, é empregado o modelo de equilíbrio de mercado simples que, permite estudar a relação entre os retornos dos ativos, do mercado e do ativo livre de risco. A derivação das equações do modelo pode ser encontrada em Wells (2013). De modo geral, pode-se definir a linha de mercado como:

$$
R_{i}=\alpha_{i}+\beta_{i} R_{M}+\varepsilon_{i}
$$

em que $\mathrm{R}_{M}$ é o excesso de retorno do mercado em relação ao ativo livre de risco; $\beta_{i}$ é o coeficiente de sensibilidade ao risco do ativo $i$ em relação ao risco do mercado como um todo; $a_{i}$ representa o componente do retorno ao ativo $i$, que é independente de $\mathrm{R}_{M}$; e $\varepsilon_{i}$ é um ruído branco.

O coeficiente beta é o parâmetro mais importante presente na equação anterior, pois determina como os retornos de cada ativo variam de acordo com o retorno do mercado, sendo, então, utilizado para mensurar o risco sistêmico (ou risco de mercado). Um valor de $\beta_{i}$ maior que um sugere que os retornos dos ativos tendem a alavancar as mudanças nos retornos de mercado e, portanto, o ativo $i$ é considerado um investimento agressivo, enquanto ativos $\operatorname{com} \beta_{i}$ menor que um são considerados investimentos mais conservadores: quanto mais próximo de zero estiver o beta, mais conservador é o ativo [1].

Em uma fase inicial dessa literatura, os coeficientes $\beta$ foram considerados invariantes no tempo. Porém, como observado por Gastaldi e Nardecchia (2003), a literatura foi evoluindo com fortes evidências de que a sensibilidade dos retornos dos ativos em relação ao mercado não era constante ao longo do tempo. A estimação do parâmetro $\beta$, que era feita de modo invariante no tempo, passou a ser refutada pela evidência empírica - como pode-se notar nas pesquisas de Brooks, Faff e Lee (1994) e Cheng (1997) -, surgindo, então, novas abordagens para a estimação do modelo considerando a possibilidade do parâmetro assumir diferentes regimes no decorrer do tempo - conforme Huang (2000) e Škrinjarić (2014) -, ou variando a cada ponto do tempo - como nos estudos realizados por Bodurtha e Mark (1991), Ferson e Harvey (1999), Gastaldi e Nardecchia (2003), Ang e Chen (2007) e Adrian e Franzoni (2009). Além de os modelos com coeficientes (mais especificamente os betas) variando a cada ponto do tempo corresponderem melhor

$1 \quad$ Podem ser consultadas em Fabozzi et al. (2007). 
à realidade, ajustaram-se melhor a problemas práticos de previsão e mensuração dos movimentos dos retornos dos ativos.

No tocante à aplicação do modelo CAPM ao mercado brasileiro, Araújo, Oliveira e Silva (2012) fazem um levantamento com as publicações em congressos relevantes e periódicos de trabalhos envolvendo o modelo com aplicações até 2008. Para o período posterior a 2008, foram realizados alguns trabalhos interessantes, como o de Mussa, Rogers e Securato (2009), que utilizam o modelo CAPM, entre outros modelos de precificação, em sua metodologia preditiva para o mercado acionário brasileiro, além dos de Flister, Bressan e Amaral (2011) e Mazzeu, Costa e Santos (2013), que tomam abordagens distintas para construir modelos CAPM condicionais. Já em uma análise comparativa, contando com os casos argentino, brasileiro e chileno, Tambosi et al. (2010) contrastam os CAPM condicionais desses países em relação ao mercado dos Estados Unidos. Por fim, Oliveira e Cunha (2017) realizam a estimação do modelo com inclusão das quebras estruturais.

\subsection{CAPM com Mudança de Regime: Markov Switching}

No modelo CAPM com mudança de regime, supõe-se que há uma especificação para estados ou regimes diferentes. Um estado é o conservador e o outro é um regime de alta sensibilidade (agressivo), ambos correspondem a estados nos quais os retornos dos ativos podem ser mais ou menos sensíveis aos retornos do mercado. Tal abordagem segue propostas semelhantes às de Huang (2000) e Škrinjarić (2014).

O modelo CAPM passa, então, a ser escrito de forma que o coeficiente de sensibilidade ao retorno de mercado possa assumir dois regimes:

$$
R_{i t}=\alpha_{i t}+\beta_{i S_{t}} R_{M t}+\varepsilon_{S_{t}}
$$

Nesse caso, o coeficiente e o termo de erro podem agora alterar seu estado ao longo do tempo, e é a variável de estado não observável, que evolui de acordo com o processo Markov switching de primeira ordem, definido como:

$$
\begin{aligned}
& P\left[S_{t}=1 \mid S_{t-1}=1\right]=p=p_{11}, P\left[S_{t}=2 \mid S_{t-1}=1\right]=1-p=p_{21} \\
& P\left[S_{t}=2 \mid S_{t-1}=2\right]=q=p_{22}, P\left[S_{t}=1 \mid S_{t-1}=2\right]=1-q=p_{12}
\end{aligned}
$$


em que $P\left[S_{t}=j \mid S_{t-1}=i\right]$ é a probabilidade de que o objeto de estudo esteja no estado $j$ em $t$, dado que esteve no regime $i$ em $t-1 ; p$ e q são as probabilidades de transição dos regimes conservador e de alta sensibilidade.

A matriz de probabilidades de transição pode ser descrita do seguinte modo:

$$
P=\left[\begin{array}{ll}
p_{11} & p_{12} \\
p_{21} & p_{22}
\end{array}\right]
$$

Como se tratam de probabilidades, $0 \leq \mathrm{p}_{i j} \leq 1$ e $\forall j$ e $\sum_{j=1}^{2} p_{i j}=1$.

Assumindo $\alpha_{\mathrm{it}}=0$, os coeficientes podem ser divididos em dois regimes:

$$
\beta_{i S_{t}}=\left\{\begin{array}{lll}
\beta_{i}^{1}, & \text { se } & S_{t}=1 \\
\beta_{i}^{2}, & \text { se } & S_{t}=2
\end{array} .\right.
$$

O termo de erro $\varepsilon_{S_{t}}$ é independente e distribuído identicamente, com distribuição $\mathcal{N}\left(0, \sigma_{S_{t}}^{2}\right)$, de modo que:

$$
\sigma_{S_{t}}=\left\{\begin{array}{lll}
\sigma_{i 1}, & \text { se } & S_{t}=1 \\
\sigma_{i 2}, & \text { se } & S_{t}=2
\end{array}\right.
$$

Uma vez definido o intercepto igual a zero, tomando-se esperança da equação 2, chega-se a:

$$
E\left(R_{i t}\right)=\beta_{i S_{t}} E\left(R_{M t}\right)
$$

Para a estimação dos parâmetros do modelo com mudança de regime, denota-se: $\mathrm{y}_{t}=\mathrm{R}_{t}, \mathrm{x}_{\mathrm{t}}=\left(1, \mathrm{R}_{\mathrm{Mt}}\right), \phi_{S t}=\left(\alpha_{i t}, \beta_{i S_{t}}\right)^{\prime}$. O modelo pode ser escrito como:

$$
y_{t}=x_{t}{ }^{\prime} \phi_{S t}+\varepsilon_{t}
$$

Além disso, $\mathrm{X}_{\mathrm{t}}=\left(\mathrm{x}_{1}, \mathrm{X}_{2}, \ldots, \mathrm{x}_{\mathrm{t}}\right)^{\prime}$ e $\mathrm{Y}_{\mathrm{t}}=\left(\mathrm{y}_{1}, \mathrm{y}_{2}, \ldots, \mathrm{y}_{\mathrm{t}}\right)^{\prime}$ representam toda a informação da amostra em relação a sua respectiva variável. O vetor $\theta=\left(p, q, \beta_{i}^{1}, \beta_{i}^{2}, \sigma_{i 1}, \sigma_{i 2}\right)$ representa os parâmetros desconhecidos a serem estimados. Se o regime é conhecido, a função de verossimilhança condicionada ao determinado regime é descrita a seguir:

$$
f\left(y_{t} \mid S_{t} ; X_{t}, \theta\right)=\frac{1}{\sqrt{2 \pi} \sigma} \exp \left[-\frac{\left(y_{t}-x_{t}{ }^{\prime} \phi_{S t}\right)}{2 \sigma^{2}}\right]
$$


Assim, é aplicada a estimação por máxima verossimilhança para a resolução do problema. Os procedimentos de filtragem e suavização para obtenção dos parâmetros podem ser encontrados nos estudos de Shen (1994) e Huang (2000).

\subsection{Modelo de Mercado com Filtro de Kalman}

Há vários estudos envolvendo aplicações do modelo CAPM os quais argumentam que uma falha na aplicação do modelo poderia ser atribuída à hipótese de que o risco de mercado seria constante ao longo do tempo. Ao se confrontar essa hipótese, tem-se a versão condicional do CAPM. Nesse caso, o modelo é interpretado como um modelo de fator dinâmico e pode ser escrito em formato estado-espaço. Com essa formulação, os parâmetros podem ser estimados recursivamente através do algoritmo do filtro de Kalman

O filtro de Kalman consiste em um conjunto de equações que torna possível a estimação e atualização das estimativas à medida que novas observações da variável de interesse são conhecidas. Dessa forma, os coeficientes ou parâmetros vão sendo atualizados ao longo do tempo em um processo de ajuste. O processo de filtragem é dividido em duas partes, a primeira trata da previsão e a segunda consiste na atualização, que permitem atualizar a previsão da variável de estado a cada nova observação disponível em cada ponto do tempo. Além disso, através do processo de suavização é possível basear a estimação dos estados em todas as observações disponíveis. Esse procedimento é chamado de suavizador de Kalman.

O modelo apresentado na equação 1 pode ser representado em formato estado-espaço como:

$$
\begin{gathered}
R_{i t}=\alpha_{i t}+\beta_{i t} R_{M t}+\varepsilon_{i t}, \quad \varepsilon_{i t} \sim N\left(0, \sigma_{\varepsilon_{i}}^{2}\right) \\
\alpha_{i t}=\alpha_{i, t-1}+v_{t}, \quad v_{i t} \sim N\left(0, \sigma_{v_{i}}^{2}\right) \\
\beta_{i t}=\beta_{i, t-1}+\eta_{t}, \quad \eta_{i t} \sim N\left(0, \sigma_{\eta_{i}}^{2}\right)
\end{gathered}
$$

Nesse caso, os termos de erro $\left(\varepsilon_{i t}, v_{i t} e \eta_{i t}\right)$ são, por hipótese, gaussianos e mutuamente independentes. A equação de transição (13) é o processo que governa a dinâmica do beta. Assume-se que $\gamma=1$ e que a equação de transição para 
o beta é um processo do tipo passeio aleatório. Uma hipótese similar é feita para o parâmetro alfa, que também deve seguir um

O sistema pode ser escrito numa notação mais compacta como:

$$
\begin{aligned}
& R_{t}=C_{t} X_{t}+\varepsilon_{t}, \\
& X_{t}=\Gamma X_{t-1}+\xi_{t},
\end{aligned}
$$

em que $C_{t}=\left[1, R_{M t}\right], X_{t}=\left[\alpha_{t}, \beta_{t}\right]^{\prime}$ é o vetor de estados e $\xi_{t}=\left[\eta_{t}, v_{t}\right]^{\prime}$ representa os termos de erro do vetor autorregressivo (VAR) dos estados, dado que o processo que governa a dinâmica dos estados é um passeio aleatório, $\Gamma=1$. Para a inicialização das recursões do filtro de Kalman, assume-se a seguinte condição inicial para os estados:

$$
\begin{aligned}
& \beta_{0} \sim N\left(\beta_{0}, P_{0}\right) \\
& \alpha_{0} \sim N\left(\alpha_{0}, P_{0}^{\prime}\right)
\end{aligned}
$$

Os parâmetros do sistema $\theta_{i}=\left(\sigma_{\varepsilon_{i^{\prime}}}^{2}, \sigma_{\eta_{i}}^{2}, \sigma_{v_{i}}^{2}\right)$ são estimados por otimização numérica, maximizando a função de verossimilhança:

$$
\log \mathcal{L}\left(\theta_{i}\right)=-\frac{T}{2} \log (2 \pi)-\frac{1}{2} \sum_{t=1}^{T} \log \left(f_{i, t}\left(\theta_{i}\right)\right)-\frac{1}{2} \sum_{t=1}^{T} \frac{v_{i t}^{2}\left(\theta_{i}\right)}{\log \left(f_{i, t}\left(\theta_{i}\right)\right)}
$$

em que $v_{t}$ denota o erro de previsão um passo à frente e $f_{i t}$ é a variância do erro de previsão, $\operatorname{Var}\left(v_{i t}^{2}\left(\theta_{i}\right)\right)$.

Ainda, para avaliar a precisão das previsões do filtro se recorre ao valor médio absoluto do erro de previsão (mean absolute forecasting error - MAE) e ao erro médio quadrado de previsão (mean square forecasting error - MSE) como forma de mensurar a acurácia do filtro de Kalman. Essas medidas de desempenho podem ser consultadas nos trabalhos de Brooks, Faff e McKenzie (1998), McKenzie, Brooks e Faff (2000) e Gastaldi e Nardecchia (2003). 
Além do filtro de Kalman, realiza-se a estimação dos coeficientes variando ao longo do tempo com a suavização. Esse procedimento é construído de modo bem semelhante ao filtro, porém sua elaboração conta com informações referentes a toda amostra, e sua implementação é feita em sentido inverso, do fim para o ínicio da amostra. De outro modo, segundo Harvey e Stock (1993), quando se deseja estimar o vetor de estados para uma série de observações $\mathrm{Y}_{\mathrm{s}}=\mathrm{y}_{1}, \ldots, \mathrm{y}_{\mathrm{s}}$, no período $s$. Quando $s<$ t, trata-se de uma abordagem de previsão. A filtragem ocorre quando $s=t$. Por fim, lida-se com um problema de suavização quando $s>t$. A suavização traz ganhos às estimações, uma vez que considera toda a informação da amostra.

Detalhes acerca da solução computacional da função de verossimilhança, dos passos das abordagens de filtragem e da suavização de Kalman podem ser encontrados nos estudos de Gastaldi e Nardecchia (2003), Campagnoli, Petrone e Petris (2009) e Zeng e Wu (2013).

\section{Base de Dados e Análise Empírica}

Uma vez realizadas as considerações acerca da importância de se conhecer o processo de formação dos retornos dos índices setoriais, de sustentabilidade e governança, leva-se em conta o gestor ou investidor que necessita de informações para sua tomada de decisões. Além da construção do modelo CAPM com diferentes abordagens econométricas, parte-se, então, para a aplicação da metodologia descrita para o caso especial dos índices citados.

Figura 1 - Séries Ibovespa e CDI: 252 dias

1) CDI - 252 dias (\%)

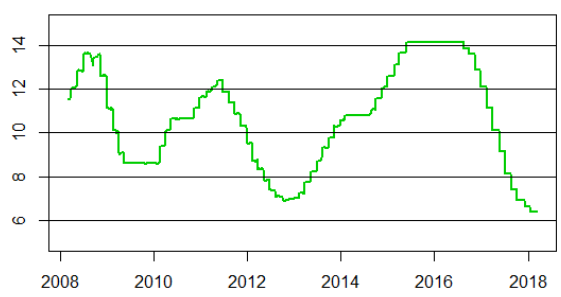

2) Ibovespa

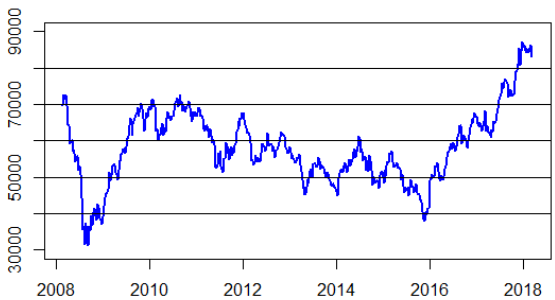

Fonte: Elaboração própria a partir de dados da base Economatica (2018). 
Figura 2 - Índices Bovespa

1) Energia Elétrica

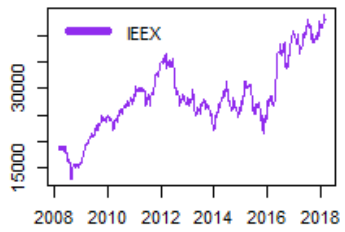

4) Imobiliário

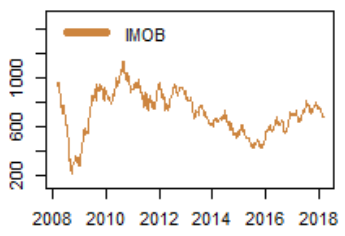

7) Utilidade Pública

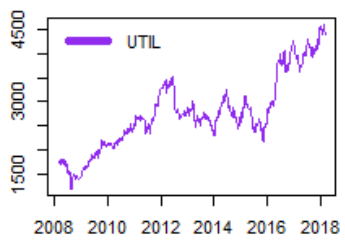

2) Setor Industrial

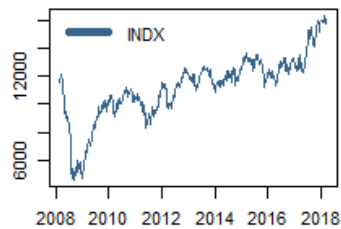

5) Financeiro

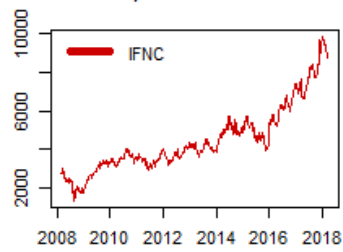

8) Sustentabilidade Empresarial

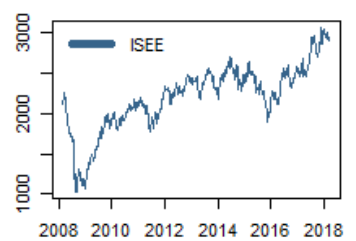

3) Consumo

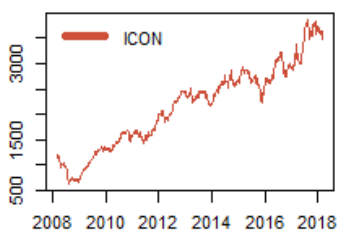

6) Materiais Básicos

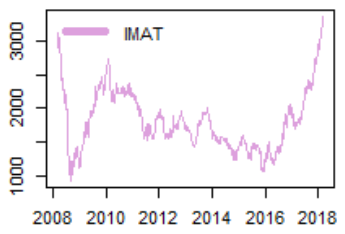

9) Governança Corporativa

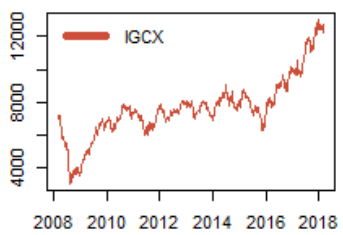

Fonte: Elaboração própria a partir de dados da base Economatica (2018).

\subsection{Base de Dados}

No presente estudo, são utilizados alguns índices que refletem o comportamento de carteiras teóricas elaboradas com ativos da B3, aos quais se recorre para a estimação dos betas em relação ao mercado brasileiro. Além disso, utiliza-se o índice Ibovespa para representar o mercado brasileiro - então sua série de retornos é $\mathrm{R}_{\mathrm{Mt}}$ no modelo - e toma-se a série diária anualizada do CDI como proxy para o ativo livre de risco.

Os índices utilizados como ativos na avaliação empírica podem ser divididos por setores industriais, indicadores de sustentabilidade e de governança corporativa. Coletam-se dados referentes aos retornos semanais de todos os índices para os últimos 10 anos, de 9 de maio de 2008 a 11 de maio de 2018. Como a elaboração dos índices é recente, opta-se por utilizar um período no qual havia observações completas do retornos de todos. Além disso, a utilização de retornos semanais é prática comum em artigos com periodicidade semelhante na área de finanças, 
como pode ser notado no trabalho de Gastaldi e Nardecchia (2003). A descrição mais detalhada de tais índices é assim feita:

1. Índices setoriais: desenvolvidos para os principais setores da economia, permitem uma análise segmentada do mercado:

a) índice de energia elétrica (IEEX): mede o comportamento das empresas mais importantes do setor de energia elétrica; foi o primeiro índice setorial elaborado pela bolsa de valores brasileira;

a) índice do setor industrial (INDX): elaborado em parceria com a Federação das Indústrias do Estado de São Paulo (Fiesp), é um indicador do desempenho médio das ações mais representativas do setor industrial;

c) índice de consumo (Icon): tem em sua composição as principais empresas do setor de consumo;

d) índice imobiliário (Imob): composto por papéis de empresas da construção civil, intermediação imobiliária e afins;

e) índice financeiro (IFNC): representa o setor de intermediários e serviços financeiros, além de previdência e seguros;

f) índice de materiais násicos (Imat): constituído por papéis das empresas mais líquidas e representativas do setor de materiais básicos;

g) índice de utilidade pública (Util): representa empresas que prestam serviços de utilidade pública - energia elétrica, fornecimento e distribuição de gás, abastecimento de água e saneamento.

2. Índices de sustentabilidade e governança: compostos por empresas que adotam práticas mais transparentes e compromissadas com seus acionistas, além de se pautarem pela responsabilidade socioambiental e seguirem práticas de governança corporativa:

a) índice de sustentabilidade empresarial (Isee): formado por empresas que adotam ações com vistas à eficiência econômica, equilíbrio socioambiental, justiça social e demais práticas sustentáveis;

b) índice de ações com governança corporativa (IGCX): composto por uma carteira formada com papéis de empresas que apresentam bons indicadores de governança corporativa.

\subsection{Coeficientes no Modelo Estático}

Antes da estimação do modelo CAPM com Markov switching e filtro de Kalman, realiza-se a estimação do modelo com os coeficientes constantes no tempo. Para tanto, são feitas estimações por mínimos quadrados ordinários (MQO), cujos resultados para o modelo em seu formato estático são apresentados a seguir. 
A Tabela 1 apresenta os valores dos coeficientes estimados para o modelo CAPM estático, além do coeficiente de determinação 0 e nível de significância.

Tabela 1 - Coeficientes de regressão simples

\begin{tabular}{llllll}
\hline Índice & $\alpha_{i}$ & $p$-valor & $\beta_{i}$ & $p$-valor & $R_{i} 2$ \\
\hline IEEX & 0,0005 & 0,5812 & 0,5354 & $<0,0001^{* * *}$ & 0,454 \\
INDX & 0,00006 & 0,203 & 0,7965 & $<0,0001^{* * *}$ & 0,777 \\
ICON & 0,0011 & $0,0964^{*}$ & 0,6313 & $<0,0001^{* * *}$ & 0,633 \\
IMOB & $-0,0008$ & 0,4950 & 1,0904 & $<0,0001^{* * *}$ & 0,657 \\
IFNC & 0,0019 & $0,0134^{* *}$ & 1,0808 & $<0,0001^{* * *}$ & 0,824 \\
IMAT & $-0,00009$ & 0,9390 & 1,0090 & $<0,0001^{* * *}$ & 0,623 \\
UTIL & 0,0002 & 0,4670 & 0,5639 & $<0,0001^{* * *}$ & 0,430 \\
ISEE & $-0,00001$ & 0,8332 & 0,8332 & $<0,0001^{* * *}$ & 0,877 \\
IGCX & 0,0005 & 0,1250 & 0,8619 & $<0,0001^{* * *}$ & 0,944 \\
\hline
\end{tabular}

Fonte: Elaborado própria a partir do software $R$.

Os valores dos $\alpha$, além de muitos pequenos, são, na maioria da vezes, estatisticamente não significativos. Resultados semelhantes aos encontrados por Araújo, Fajardo e Di Tavani (2006), que também obtiveram valores baixos e estatisticamente não significantes para esses parâmetros, considerando suas carteiras teóricas dividas em setores, formadas com ativos da bolsa brasileira, enquanto todos os apresentaram um alto nível de significância estatística, com $p$-valor abaixo de 0,0001. O índice com retorno mais sensível ao retorno de mercado foi o Imob: seus retornos respondem em média a 1,0904 das variações nos retornos do índice de mercado, o que é característica, segundo Gastaldi e Nardecchia (2003) e Campagnoli, Petrone e Petris (2009), de um setor com empresas mais alavancadas ou agressivas em relação ao mercado como um todo. Já o índice que apresentou menor sensibilidade ao mercado foi o Ieex, com seu beta assumindo 0,5354 no modelo estático, caracterizando, segundo os autores anteriormente citados, um setor mais conservador se comparado ao restante do mercado.

Em relação ao ajuste da regressão, o IGCX apresentou maior coeficiente de determinação $(0,944)$, o que significa dizer que 94,40 dos retornos da carteira teórica formada por ativos de empresas com boas práticas de governança são explicados pelo retorno de mercado. Por outro lado, o índice de utilidade pública (Util) teve o menor $(0,430)$, isto é, em média $43 \%$ das variações nos retornos do setor de utilidade pública pode ser explicado pelo retorno do Ibovespa. Além disso, cabe ressaltar que os resíduos das regressões não apresentaram autocorrelações significativas para nenhum dos ativos analisados.

Esses coeficientes obtidos por MQO dão um cenário do comportamento médio da sensibilidade do retorno dos ativos em relação ao retorno do mercado para o período analisado e possibilitam a comparação com os valores dos 
modelos com coeficientes variando no tempo, obtidos com as demais técnicas de estimação.

\subsection{Betas com Mudança de Regime: Markov Switching}

Os resultados da estimação do modelo CAPM com a abordagem Markov switching são apresentados na Tabela 2.

Tabela 2 - Resultados da estimação com Markov switching

\begin{tabular}{lllllllllll}
\hline & Indice & $\mathrm{p}$-valor & $\sigma_{i 1}$ & & & $\mathrm{p}$-valor & $\sigma_{\mathrm{i} 2}$ & & $p_{i}$ & $q_{i}$ \\
\hline Ieex & 0,5619 & $<0,0001$ & 0,0169 & 0,5034 & 0,4541 & $<0,0001$ & 0,0465 & 0,3754 & 0,9851 & 0,8131 \\
INDX & 0,9578 & $<0,0001$ & 0,0124 & 0,9014 & 0,4832 & $<0,0001$ & 0,0134 & 0,5552 & 0,9909 & 0,9861 \\
Icon & 0,8118 & $<0,0001$ & 0,0118 & 0,8776 & 0,5511 & $<0,0001$ & 0,0147 & 0,6526 & 0,1464 & 0,7044 \\
Imob & 1,3219 & $<0,0001$ & 0,0452 & 0,7627 & 0,9338 & $<0,0001$ & 0,0227 & 0,5988 & 0,9992 & 0,9977 \\
IFNC & 1,3392 & $<0,0001$ & 0,0186 & 0,8974 & 0,8612 & $<0,0001$ & 0,0125 & 0,8400 & 0,4159 & 0,6910 \\
Imat & 1,2062 & $<0,0001$ & 0,0196 & 0,8261 & 0,4816 & $<0,0001$ & 0,0365 & 0,1859 & 0,9761 & 0,9324 \\
Util & 0,6910 & $<0,0001$ & 0,0187 & 0,5998 & 0,0437 & 0,7255 & 0,0357 & 0,1443 & 0,9731 & 0,7557 \\
Isee & 0,9712 & $<0,0001$ & 0,0155 & 0,9271 & 0,7314 & $<0,0001$ & 0,0088 & 0,8588 & 0,9999 & 0,9977 \\
IGCX & 0,9078 & $<0,0001$ & 0,0064 & 0,9640 & 0,6861 & $<0,0001$ & 0,0072 & 0,9016 & 0,9822 & 0,9508 \\
\hline
\end{tabular}

Fonte: Elaboração própria a partir do software R-Studio.

Os interceptos do modelo não foram estatisticamente significativos assim como ocorreu na estimação por MQO. A análise, então, se concentra nos coeficientes betas, seu poder de determinação e nas probabilidades do CAPM estar em um regime de maior ou menor sensibilidade dos retornos dos índices em relação ao mercado. Além disso, são apresentados valores referentes ao desvio padrão do termo de erro de cada regime e ao coeficiente de determinação.

Na Tabela 2, ainda é possível observar as probabilidades de manutenção de estado denotadas por e para cada ativo, ao passo que e denotam a probabilidade de alteração de estado a cada ponto do tempo. A Figura 3 traz as probabilidades filtradas e suavizadas do modelo estar em um regime de alto ou baixo risco sistêmico. Pode-se notar que os índices financeiro e de consumo são os com maior probabilidade de mudança de regime no decorrer do tempo. Por outro lado, os índices do setor imobiliário e de sustentabilidade empresarial, a partir do momento em que fazem a transição de regimes, têm probabilidades maiores de permanecer no regime posterior. 
Figura 3 - Probabilidades entre regimes: Markov switching
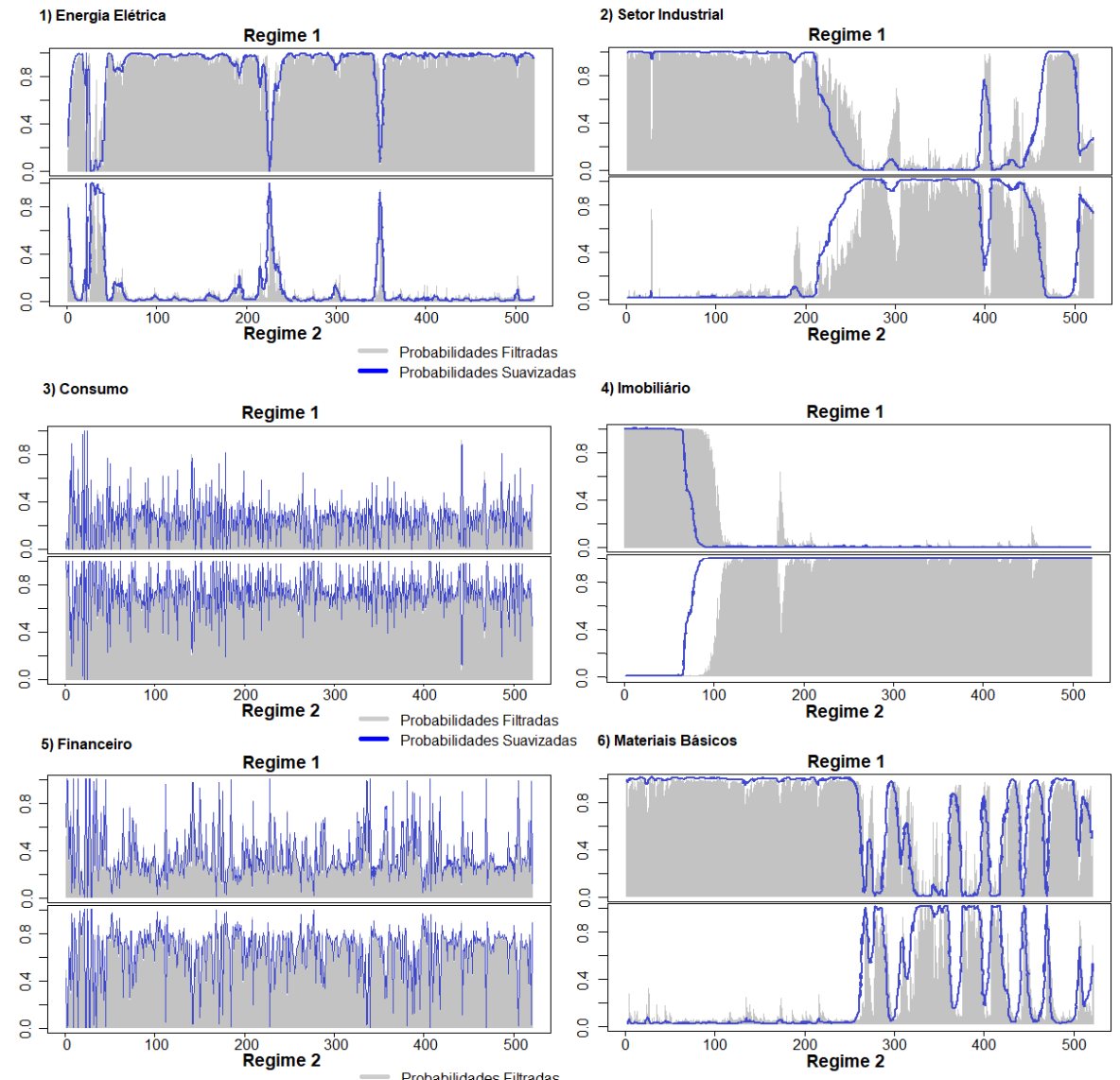

7) Sustentabilidade Empresarial

Regime 1

Regime 1
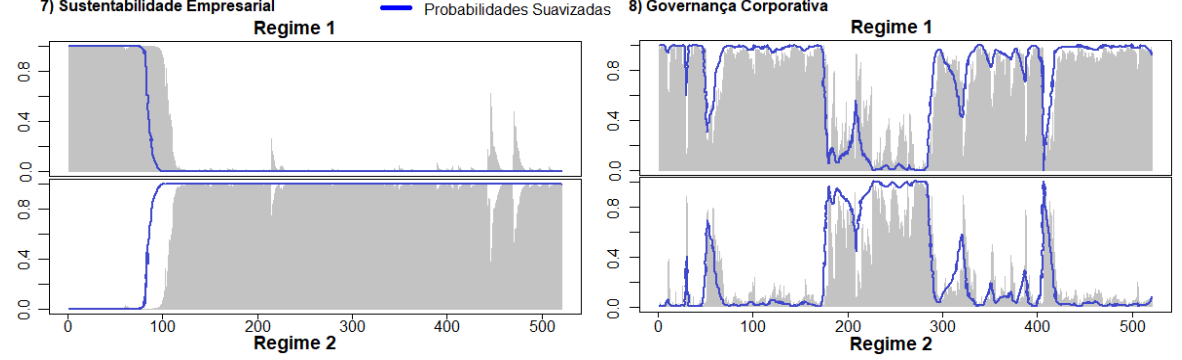

Fonte: Elaboração própria a partir do software R. 
À exceção do índice Util, todos os demais índices apresentaram os coeficientes de sensibilidade ao mercado estatisticamente significativos com no mínimo 99\% de confiança para os dois regimes. O Imat foi o índice que apresentou maior diferença entre os $\beta$ dos regimes de alta e baixa sensibilidade ao mercado. Do ponto de vista econômico, isso significa dizer que o índice alternou de um regime de alavancagem ou agressivo, com $\beta=1,2062$, para uma regime mais conservador, $\beta=0,4816$, durante o período analisado. Quando se analisa o gráfico das probabilidades de mudança de regime desse índice, nota-se que as probabilidades desse ativo estar no regime de alavancagem foram grandes na primeira metade da amostra, enquanto houve um quadro de alternância entre regimes na parte final da amostra.

Enquanto o índice do setor elétrico teve menor diferença entre os coeficientes dos dois regimes, com o beta do regime um sendo igual a 0,9712 e o do segundo regime, igual a 0,7314 , nota-se, analisando-se o comportamento das probabilidades filtradas e suavizadas para esse índice, que, no decorrer do período, há chances maiores de se estar no primeiro regime e a probabilidade de permanência também é alta $(98,51 \%)$.

Ainda, quando se analisam os regimes aos quais os retornos dos índices têm maior probabilidade de permanecer a maior parte do período, o Ieex e o Imat, claramente, têm maior chance de ficarem no regime de maior sensibilidade aos retornos do mercado, isto é, permanecerem em situação de alavancagem ou num regime mais agressivo do ponto de vista econômico. Já os retornos do Imob e Isee mostraram um comportamento no qual foi mais provável permanecerem num regime de menor risco sistêmico a maior parte da amostra, ou seja, um comportamento mais conservador, segundo a teoria econômica. No caso destes últimos índices, pode-se notar a maior probabilidade de permanência no regime de menor risco de mercado nos oito anos finais do período.

Em suma, a análise desses gráficos permite, a cada ponto de tempo, avaliar a probabilidade de que os retornos de qualquer um dos índices esteja em um regime de alta ou baixa sensibilidade ao mercado. Conforme Gastaldi e Nardecchia (2003) e Campagnoli, Petrone e Petris (2009), podem ser definidos também como regimes de alavancagem (agressivo) ou conservadores para o caso de betas baixos.

\subsection{Resultados com a Filtragem e Suavização de Kalman}

A implementação do filtro de Kalman foi realizada conforme a metodologia, utilizando-se procedimentos computacionais para a estimação dos parâmetros desconhecidos por máxima verosimilhança.6 As demais etapas do algorítimo do filtro de Kalman e os resultados das medidas de precisão, previsão dos coeficientes um passo à frente da amostra, betas medianos, médios e máximos são apresentados na Tabela 3. 
Tabela 3 - Descrição da estimação via filtro de Kalman

\begin{tabular}{lccccccc}
\hline Índice & $\mathbf{M A E}_{i}$ & $\mathbf{M S E}_{i}$ & $\beta_{i}, T+\mathbf{1}$ & $\beta_{i}$-mediano & $\beta_{i}$-médio & $\beta_{i}$-máximo & Variância $\left(\beta_{i}\right)$ \\
\hline Ieex & 0,01566 & 0,000492 & 0,4602 & 0,5890 & 0,5596 & 1,3307 & 0,084155 \\
INDX & 0,01029 & 0,000212 & 0,6915 & 0,7429 & 0,7545 & 1,4177 & 0,062601 \\
Icon & 0,01253 & 0,000284 & 0,7007 & 0,6134 & 0,6269 & 1,2930 & 0,032279 \\
Imob & 0,02092 & 0,000804 & 0,8872 & 0,9902 & 1,0222 & 1,4418 & 0,030154 \\
IFNC & 0,01310 & 0,000349 & 1,3621 & 1,0724 & 0,9718 & 1,5722 & 0,035654 \\
Imat & 0,01953 & 0,000772 & 0.7177 & 1,0184 & 1,0256 & 1,4904 & 0,107735 \\
Util & 0,01720 & 0,000605 & 0,3991 & 0,6343 & 0,5991 & 1,2061 & 0,092685 \\
Isee & 0,00803 & 0,000117 & 0,8806 & 0,7658 & 0,7742 & 1,0707 & 0,014375 \\
IGCX & 0,00554 & 0,000617 & 0,8680 & 0,8461 & 0,8290 & 1,1536 & 0,015356 \\
\hline
\end{tabular}

Fonte: Elaboração própria a partir do software $R$.

Os resultados dos coeficientes $\alpha$, assim como os do modelo estático, foram estatisticamente insignificantes. Então, optou-se por omitir os $\alpha$ do restante da análise. Além do mais, nesse tipo de problema o grande foco de estudo dos modelos CAPM concentra-se no comportamento dos parâmetros $\beta$, o que de fato é de interesse do investidor avaliar como se comportou um setor ou um conjunto de empresas em relação ao mercado para um período de avaliação. Cabe ressaltar que essa abordagem permite a previsão um passo à frente do risco sistêmico para cada índice, o que possibilita ao agente ter um norteamento em relação ao impacto do retorno do mercado em determinado setor ou conjunto de empresas que adote práticas sustentáveis e de governança utilizadas na construção dessas carteiras, no tempo posterior à amostra. Isso pode ser aplicado em cada momento no qual haja novas observações da amostra.

Ao analisar-se os betas dos ativos (tomando os índices como tais), pode-se notar que realmente eles não são constantes no tempo. Além de variarem no tempo, alguns são muito voláteis, indicando que a sensibilidade do retorno dos índices em relação ao retorno do mercado pode ser muito dispersa no tempo. Em alguns casos, como o do índice do setor elétrico, houve variações dos betas no intervalo entre -0,5 e 1,4. No entanto, esse setor pode ter apresentado comportamento atípico devido à interferência do governo na política de preços das empresas que o compõem, especialmente em 2012, quando se forçou o setor a adotar um corte de tarifas de cerda de $20 \%$, gerando distorções no setor.

Ainda, o beta que atingiu maior valor, representando o ponto de maior sensibilidade do retorno de um ativo em relação ao mercado, foi o do IFNC, que atingiu o valor de 1,5722 em uma das observações mais recentes, isto é, esteve mais alavancado ou num regime mais agressivo. O Ieex chegou apresentar períodos de sensibilidade negativa durante a mudança de política de preços da energia elétrica em 2012, fato que não tem aderência na teoria econômica, porém é resultado pro- 
vável da forte interferência do governo no período em que ocorreu: os retornos do setor passaram a responder provavelmente a fatores políticos, e não ao índice de mercardo.

Figura 4 - Betas dos Índices Bovespa com a suavização de Kalman

1) Setor Elétrico

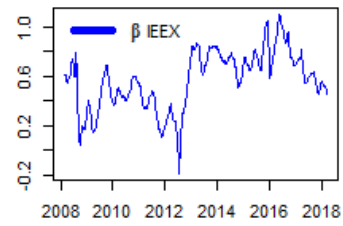

4) Imobiliário

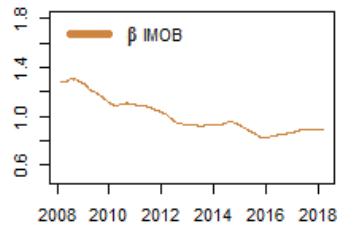

7) Utilidade Pública

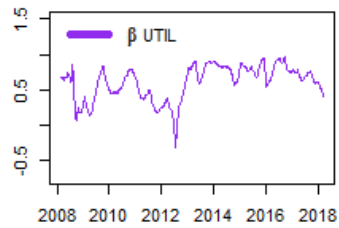

2) Setor Industrial

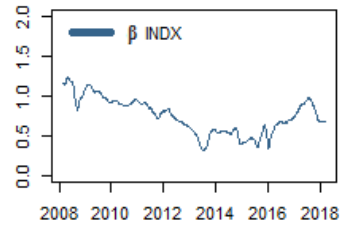

5) Financeiro

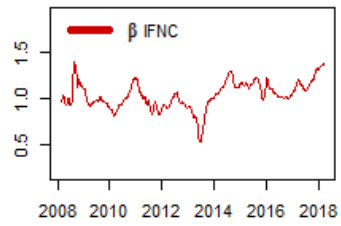

8) Sustentabilidade Empresarial

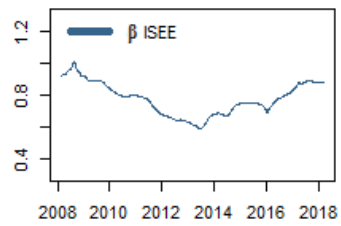

3) Consumo

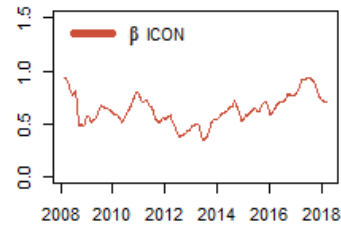

6) Materiais Básicos

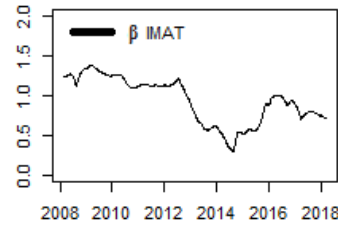

9) Governança Corporativa

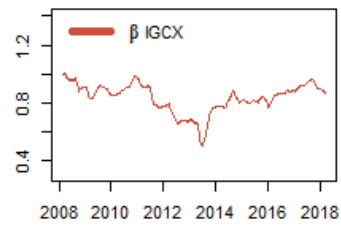

Fonte: Elaboração própria a partir do software R.

Ao analisar os betas dos ativos (tomando os índices como tais), pode-se notar que realmente eles não são constantes no tempo. Além de variarem no tempo alguns são muito voláteis, indicando que a sensibilidade do retorno dos índices em relação ao retorno do mercado pode ser muito dispersa no tempo. Em alguns casos, como o do índice do setor elétrico, houve variações dos betas no intervalo entre -0,5 e 1,4. No entanto, este setor pode ter apresentado comportamento atípico devido à interferência do governo na política de preços das empresas que o compõem, especialmente em 2012, quando se forçou o setor a adotar um corte de tarifas de cerda de $20 \%$, gerando distorções no setor.

Ainda, o beta que atingiu maior valor, representando o ponto de maior sensibilidade do retorno de um ativo em relação ao mercado foi o do IFNC que atingiu o valor de 1,5722 em uma das observações mais recentes, isto é, esteve mais alavancado ou num regime mais agressivo. Enquanto o IEEX chegou apresentar 
períodos de sensibilidade negativa durante a mudança de política de preços da energia elétrica em 2012, fato que não tem aderência na teoria econômica, porém é resultado provável da forte interferência do governo no período em que ocorreu, os retornos do setor passaram a responder provavelmente a fatores políticos e não ao índice de mercado.

O Imat apresentou o maior beta médio, 1,0256, indicando maior sensibilidade em média aos retornos do mercado, enquanto o Ieex apresentou o menor beta médio, ou seja, é menos sensível em média a variações no retorno do mercado. A série de $\beta$ do Imat teve a maior variância $(0,107735)$, já a série do Isee apresentou a menor variância $(0,014375)$, indicando menor dispersão dos betas ao longo do período analisado.

A Tabela 4 apresenta algumas estatísticas dos $\beta$ estimados via suavizador de Kalman.

Tabela 4 - Estatísticas beta estimadas via suavizador de Kalman

\begin{tabular}{lccccc}
\hline Índice & $\beta_{i}$-mínimo & $\beta_{i}$-mediano & $\beta_{i}$-médio & $\beta_{i}$-máximo & Variância $\left(\beta_{i}\right)$ \\
\hline Ieex & $-0,1908$ & 0,5947 & 0,5677 & 1,0962 & 0,0557 \\
INDX & 0,3144 & 0,7375 & 0,7494 & 1,2417 & 0,0503 \\
Icon & 0,3444 & 0,6199 & 0.6313 & 0,9405 & 0,0188 \\
Imob & 0,8196 & 0,9415 & 1,0017 & 1,3152 & 0,0196 \\
IFNC & 0,5352 & 1,0193 & 1,0371 & 1,3981 & 0,0214 \\
Imat & 0,2996 & 0,9963 & 0,9421 & 1,3919 & 0,0835 \\
Util & $-0,3328$ & 0,6678 & 0,6032 & 0,9607 & 0,0588 \\
Isee & 0,5914 & 0,7782 & 0,7769 & 1,0118 & 0,0103 \\
IGCX & 0,5017 & 0,8550 & 0,8325 & 1,0032 & 0,0097 \\
\hline
\end{tabular}

Fonte: Elaboração própria a partir do software R.

Para os índices setoriais de energia elétrica e utilidade pública (que também engloba o setor de energia elétrica), houve novamente betas negativos, provavelmente devido à mudança de preços relatada anteriormente. Os índices que apresentaram maior beta médio foram o financeiro e o imobiliário (inclusive acima de um), indicando que os retornos desses setores reagem em média mais agressivamente a variações no retorno do mercado, setores que seriam mais alavancados do ponto de vista teórico. Por outro lado, o índice do setor elétrico teve o menor beta médio, porém com alta dispersão dos valores do coeficiente no decorrer do período, consequência provável da interferência governamental no setor, que gerou distorções já relatas anteriormente.

Além disso, pelas técnicas de filtragem e suavização fica evidente, como no caso do índice setorial imobiliário, que houve uma forte mudança no coeficiente de sensibilidade ao retorno de mercado, corroborando os resultados obtidos na análise com mudança de regime. Este coeficiente decai de valores, para perío- 
dos iniciais, acima de 1,3, para valores abaixo de 0,9, em períodos mais recentes, revelando uma mudança no padrão da sensibilidade dos seus retornos. Há uma diminuição na alavancagem do setor, pois, de outro modo, torna-se menos agressivo em relação ao mercado. Deve-se levar em conta a grave crise enfrentada, recentemente, pelo setor devido à recessão e aos problemas judiciais enfrentados pelas empresas que o compõe, que podem ter afetado os resultados dos $\beta$, tornando seus resultados mais distantes do mercado.

\section{Considerações Finais}

O presente artigo levantou uma pequena discussão acerca da importância, do ponto de vista do gestor ou investidor institucional, de se conhecer o comportamento dos retornos dos índices setoriais ou de índices compostos por empresas com boas práticas corporativas, além apresentar o modelo CAPM e suas estimações pelas abordagens Markov switching e filtro/suavizador de Kalman. Discorreu-se brevemente sobre a evolução dos modelos CAPM e algumas aplicações ao caso brasileiro. Em seguida, tomou-se a linha de mercado em uma abordagem temporal, tornando possível a estimação os coeficientes do modelo CAPM com mudanças de regime e coeficientes variando a cada ponto do tempo a partir do filtro e suavização de Kalman.

Em um primeiro momento, foram estimados os coeficientes do modelo de forma estática. Nesse caso, foram obtidos alguns resultados interessantes, como os de que os coeficientes alfas são em sua maioria não significativos do ponto de vista estatístico, o que é comum na literatura, como pode ser visto nos trabalhos de Gastaldi e Nardecchia (2003) e Araújo, Fajardo e Di Tavani (2006). Os maiores betas foram encontrados para os retornos dos índices do setor financeiro e imobiliário, setores que, por característica, são mais alavancados, enquanto os menores betas foram relativos aos índices do setor elétrico e de utilidade pública. O maior coeficiente de determinação evidenciado na análise foi o do IGCX, indicando que 94,4\% das variações dos retornos desse índice advêm das variações do retorno de mercado. Já o leex teve um $\mathrm{R}^{2}$ de 0,454 , denotando o menor percentual de variação de retorno de um ativo explicado pela variação dos retornos do Ibovespa em toda a análise estática.

A aplicação da abordagem Markov switching trouxe a evidência empírica de que os retornos dos índices seguem regimes distintos no decorrer do tempo - à exceção do índice de utilidade pública, no qual o segundo regime não foi estatisticamente significativo -, de modo que foi possível estruturar o risco sistêmico em dois estados, além de se atribuir probabilidades, aos retornos dos ativos, de estarem em um estado de alta/baixa sensibilidade ao retorno do mercado ou, do ponto de vista de teoria econômica, em estados de alavancagem ou conservadorismo. Os resul- 
tados mostraram que o IFNC é o ativo que tem o $\beta$ com maior probabilidade de mudança de regime, enquanto os índices Imob e Isee têm os coeficientes de risco sistêmico mais propensos a se manterem em um determinado regime, dado que estava nele no período anterior. Nesse caso, ambos permaneceram mais tempo no regime de menor sensibilidade ao mercado.

Em relação à estimação com filtro e suavizador de Kalman, nota-se que realmente os $\beta$ não são constantes ao longo do tempo. Os resultados estão de acordo com a evolução da literatura ao demonstrarem que os coeficientes de sensibilidade ao risco de mercado variam ao longo do tempo, tomando os índices setoriais, de sustentabilidade e governança como evidências para o caso brasileiro. Um ponto interessante foi a diminuição dos betas para os índices do setor imobiliário, provavelmente devido às transformações recentes das empresas do setor imobiliário listadas na bolsa e do mercado de construção civil como um todo. Outro ponto chamativo foi o resultado negativo para os betas do leex e do Util durante o período de mudanças nos preços do setor elétrico em 2012, o que não é comum à literatura de finanças e pode ter sido fruto de distorções no setor elétrico.

Por fim, cabe destacar o experimento com os índices de sustentabilidade e governança do Ibovespa, algo que, até então, não se havia realizado num contexto de modelos CAPM. Nota-se que, em todos os casos, os betas médios desses índices ficaram abaixo de um, denotando que investimentos com carteiras teóricas de empresas que compõem esses índices tendem a ser, em média, mais conservadores, além do fato de que o modelo aplicado a esses índices pode ser uma boa ferramenta de auxílio à tomada de decisões do investidor e demais agentes.

\section{Referências}

ADRIAN, T.; FRANZONI, F. Learning about beta: time-varying factor loadings, expected returns, and the conditional capm. Journal of Empirical Finance, v. 16, n. 4, p. 537-556, 2009.

ANG, A.; CHEN, J. Capm over the long run: 1926-2001. Journal of Empirical Finance, v. 14, n. 1, p. 1-40, 2007.

ARAÚJO, E.; FAJARDO, J.; DI TAVANI, L. C. Capm usando uma carteira sintética do PIB brasileiro. Estudos Econômicos, v. 36, n. 3, p. 465-505, 2006.

ARAÚJO, E. A. T.; OLIVEIRA, V. C.; SILVA, W. A. C. Capm em estudos brasileiros: uma análise da pesquisa. Revista de Contabilidade e Organizações, v. 6, n. 15, p. 95, 2012.

BACA, S. P.; GARBE, B. L.; WEISS, R. A. The rise of sector effects in major equity markets. Financial Analysts Journal, v. 56, n. 5, p. 34-40, 2000.

BLACK, F.; LITTERMAN, R. Asset allocation: combining investor views with market equilibrium. The Journal of Fixed Income Fall, v. 1, n. 2, p. 7-18, 1990. 
BODURTHA, J. N.; MARK, N. C. Testing the capm with time-varying risks and returns. The Journal of Finance, v. 46, n. 4, p. 1485-1505, 1991.

BROOKS, R. D.; FAFF, R. W.; LEE, J. H. Beta stability and portfolio formation. Pacific-Basin Finance Journal, v. 2, n. 4, p. 463-479, 1994.

BROOKS, R. D.; FAFF, R. W.; MCKENZIE, M. D. Time-varying beta risk of australian industry portfolios: a comparison of modelling techniques. Australian Journal of Management, v. 23, n. 1, p. 1-22, 1998.

CAMPAGNOli, P.; PETRONE, S.; PETRIS, G. Dynamic linear models with R. New York: Springer, 2009.

CAMPBELL, J. Y. Understanding risk and return. Journal of Political Economy, v. 104, n. 2, p. 298-345, 1996.

CHENG, J. W. A switching regression approach to the stationarity of systematic and non-systematic risks: the hong kong experience. Applied Financial Economics, v. 7, n. 1, p. 45-57, 1997.

ECONOMATICA. [S.l.: s.n.], 2018. Disponível em: https://economatica.com/. Acesso em: 10 nov. 2018.

FABOZZI, F. J. et al. Robust portfolio optimization and management. New Jersey: John Wiley E Sons, 2007.

FAMA, E. F. Risk, return and equilibrium: some clarifying comments. The Journal of Finance, V. 23, n. 1, p. 29-40, 1968.

FERSON, W. E.; HARVEY, C. R. Conditioning variables and the cross section of stock returns. The Journal of Finance, v. 54, n. 4, p. 1325-1360, 1999.

FLISTER, F. V.; BRESSAN, A. A.; AMARAL, H. F. Capm condicional no mercado brasileiro: um estudo dos efeitos momento, tamanho e book-to-market entre 1995 e 2008. Revista Brasileira de Finanças, v. 9, n. 1, 2011.

GASTALDI, M.; NARDECCHIA, A. The kalman filter approach for time-varying ß estimation. Systems Analysis Modelling Simulation, v. 43, n. 8, p. 1033-1042, 2003.

GROENEWOLD, N.; FRASER, P. Time-varying estimates of capm betas. Mathematics and Computers in Simulation, v. 48, n. 4-6, p. 531-539, 1999.

HAMILTON, J. D. A new approach to the economic analysis of nonstationary time series and the business cycle. Econometrica, v. 57, n. 2, p. 357-384, 1989.

HARVEY, A.; STOCK, J. H. Estimation, smoothing, interpolation, and distribution for structural time-series models in continuous time. In: PHILLIPS, G.; PHILLIPS, P. C. B. Models, methods and applications of econometrics. Cambridge: Wiley Blackwell, 1993. p. 55-70.

HUANG, H. C. Tests of regimes-switching capm. Applied Financial Economics, v. 10, n. 5, p. 573-578, 2000. 
HUANG, H. C. Tests of capm with nonstationary beta. International Journal of Finance $\mathbb{E}$ Economics, v. 6, n. 3, p. 255-268, 2001.

KIRBY, C.; OSTDIEK, B. It's all in the timing: simple active portfolio strategies that outperform naive diversification. Journal of Financial and Quantitative Analysis, v. 47, n. 2, p. 437-467, 2012.

LÓPEZ, M. V.; GARCIA, A.; RODRIGUEZ, L. Sustainable development and corporate performance: a study based on the Dow Jones Sustainability Index. Journal of Business Ethics, v. 75, n. 3, p. 285-300, 2007.

MACHADO, M. R.; MACHADO, M. A. V.; CORRAR, L. J. Desempenho do índice de sustentabilidade empresarial (ISE) da bolsa de valores de São Paulo. Revista Universo Contábil - FURB, v. 5, n. 2, p. 24-38, 2009.

MAZZEU, J. H. G.; COSTA, N. C. A.; SANTOS, A. A. P. Capm condicional com aprendizagem aplicado ao mercado brasileiro de ações. Revista de Administração Mackenzie, v. 14, n. 1, p. 143, 2013.

MCCOOL, S. F.; STANKEY, G. H. Indicators of sustainability: challenges and opportunities at the interface of science and policy. Environmental Management, v. 33, n. 3, p. 294-305, 2004.

MCKENZIE, M. D.; BROOKS, R. D.; FAFF, R. W. The use of domestic and world market indexes in the estimation of time-varying betas. Journal of Multinational Financial Management, v. 10, n. 1, p. 91-106, 2000.

MILANI, B. et al. Práticas de sustentabilidade, governança corporativa e responsabilidade social afetam o risco e o retorno dos investimentos? Revista de Administração da UFSM, v. 5, p. 667-682, 2012. Edição especial.

MILES, M. P.; COVIN, J. G. Environmental marketing: a source of reputational, competitive, and financial advantage. Journal of Business Ethics, v. 23, n. 3, p. 299-311, 2000.

MUSSA, A.; ROGERS, P.; SECURATO, J. R. Modelos de retornos esperados no mercado brasileiro: testes empíricos utilizando metodologia preditiva. Revista de Ciências da Administração - UFSC, v. 11, n. 23, p. 192, 2009.

OLIVEIRA, F. N.; CUNHA, F. C. S. Estimando betas de mercado com quebras estruturais. Revista Brasileira de Finanças, v. 15, n. 2, p. 252-286, 2017.

PETRIS, G. et al. State space models in R. Journal of Statistical Software, v. 41, n. 4, p. 1-25, 2011.

PLANTINGA, A.; SCHOLTENS, B. Socially responsible investing and management style of mutual funds in the Euronext stock markets. [S.1.]: Research School Systems, Organisation and Management, 2001.

REZENDE, I. A. C.; NUNES, J. G.; PORTELA, S. S. Um estudo sobre o desempenho financeiro do índice Ibovespa de sustentabilidade empresarial. Revista de Educação e Pesquisa

SHEN, C. H. Testing efficiency of the Taiwan-US forward exchange market - a Markov switching model. Asian Economic Journal, v. 8, n. 2, p. 205-216, 1994. 
ŠKRINJARIĆ, T. Testing for regime-switching CAPM on Zagreb stock exchange. Croatian Operational Research Review, v. 5, n. 2, p. 119-133, 2014.

TAMBOSI, E. et al. Teste do capm condicional dos retornos de carteiras dos mercados brasileiro, argentino e chileno, comparando-os com o mercado norte-americano. RAE-Revista de Administração de Empresas, v. 50, n. 1, 2010.

WELLS, C. The Kalman filter in finance. [S.1.]: Springer: Business Media, 2013. v. 32.

ZENG, Y.; WU, S. State-space models: applications in economics and finance. [S.1.]: Springer, 2013. V. 1 .

\section{Autor correspondente:}

Ricardo de Souza Tavares

E-mail: tavares@email.arizona.edu

Recebido em: 02/10/2018.

Aceito em: 25/09/2019.

\section{(cc) BY}

Este é um artigo de acesso aberto distribuído sob os termos da Creative Commons Attribution CC-BY 4.0, que permite uso irrestrito, distribuição e reprodução em qualquer meio, desde que o trabalho original seja devidamente citado. 